Swarthmore College

Works

$11-1-1993$

\title{
Phase Diagrams For The Blue Phases Of Highly Chiral Liquid Crystals
}

M. B. Bowling

Peter J. Collings

Swarthmore College, pcollin1@swarthmore.edu

C. J. Booth

J. W. Goodby

Follow this and additional works at: https://works.swarthmore.edu/fac-physics

Part of the Physics Commons

Let us know how access to these works benefits you

\section{Recommended Citation}

M. B. Bowling, Peter J. Collings, C. J. Booth, and J. W. Goodby. (1993). "Phase Diagrams For The Blue Phases Of Highly Chiral Liquid Crystals". Physical Review E. Volume 48, Issue 5. 4113-4115. DOI:

10.1103/PhysRevE.48.4113

https://works.swarthmore.edu/fac-physics/160

This work is brought to you for free by Swarthmore College Libraries' Works. It has been accepted for inclusion in Physics \& Astronomy Faculty Works by an authorized administrator of Works. For more information, please contact myworks@swarthmore.edu. 


\title{
Phase diagrams for the blue phases of highly chiral liquid crystals
}

\author{
Miriam B. Bowling and Peter J. Collings \\ Department of Physics and Astronomy, Swarthmore College, Swarthmore, Pennsylvania 19081 \\ Christopher J. Booth and John W. Goodby \\ School of Chemistry, The University of Hull, Hull HU6 7RX, England \\ (Received 17 June 1993)
}

\begin{abstract}
Polarizing microscopy and optical-activity measurements are used to determine the phase diagram for the blue phases of chiral-racemic mixtures of terephthaloyloxy-bis-4-(2'-methylbutyl) benzoate. Contrary to an earlier report, it is the second blue phase (BP II) rather than the first blue phase (BP I) that is not stable relative to the other blue phases at high chirality. With this development, all phase diagrams for the blue phases reported to date have the same topology. Using similar data for two other highly chiral systems, it is found that a simple scaling of the temperature and chiral-fraction axes produces phase diagrams in quantitative agreement with the present results. Thus, in spite of differences in molecular structure, the number of chiral centers, and phase-transition temperatures, these three systems possess remarkably similar phase diagrams and lend evidence for a universal phase diagram for the blue phases.
\end{abstract}

PACS number(s): 61.30.-v, 64.70.Md, 78.20.Ek

The blue phases of highly chiral liquid crystals possess orientational order that varies spatially in accordance with a crystallographic space-group symmetry. The stability of such phases relative to the chiral-nematic and isotropic phases has been demonstrated using extensive mean-field calculations of the Landau-de Gennes type. See Refs. [1] and [2] for two recent reviews of this theoretical work. The starting point for such theories is a free-energy expression, which reduces all of the properties of a liquid-crystalline system into the coefficients of the various free-energy terms. In order to generate theoretical phase diagrams, these coefficients are used to define a reduced temperature and a chirality parameter, and the free energy of each of the structures is calculated for many values of both in order to find the structure with the lowest free energy. The results indicate that as the chirality parameter is increased, first a simple cubic phase appears between the chiral-nematic and isotropic phases, then a body-centered cubic phase appears between the simple cubic phase and the chiral-nematic phase, and finally a different body-centered cubic phase appears between the simple cubic phase and the isotropic phase. Motivated by the experimental observation of a seemingly amorphous blue phase, theoretical calculations on phases with (1) only localized orientational order and (2) icosahedral symmetry have also been performed. At this point it is not clear if the amorphous blue phase corresponds to either of these structures. In all of this work, the free energy of the various liquid-crystal phases differs by only the slightest amount. It has therefore been assumed that a mean-field theory that uses only a few parameters to model a system cannot do justice to all the molecular properties that exist in nature. So while these theories produce a universal phase diagram for the blue phases, it is suspected that the wide variations in the molecular properties possible combined with the small differences in free energy between these structures will produce phase diagrams in nature that are qualitatively and certainly quantitatively different.

Phase diagrams on chiral-racemic mixtures are most likely to correspond to these theoretical phase diagrams. The first one reported was on terephthaloyloxy-bis-4-(2'methylbutyl) benzoate (TOBMB), a compound with three phenyl groups, two chiral centers, and phase-transition temperatures around $160^{\circ} \mathrm{C}$ [3]. As the chiral fraction was increased, first a blue phase, BP I (a body-centered cubic phase), appeared, then BP II (a simple cubic phase) appeared, and finally BP III (an amorphous phase) appeared. At even higher chiral fraction, it was reported that BP I became unstable relative to BP II and the chiral-nematic phase. Later, the phase diagrams for a number of chiral-racemic systems were reported, including 4-(2-methylbutyl)phenyl-4-hexyloxybenzoate (CE4), a compound with two phenyl groups, one chiral center, and phase-transition temperatures near $40^{\circ} \mathrm{C}$, and $4^{\prime \prime}-(2-$ methylbutylphenyl)-4'-(2-methylbutyl)-4-biphenylcarboxylate (CE2), a compound with one biphenyl group and one phenyl group, two chiral centers, and phasetransition temperatures around $120^{\circ} \mathrm{C}$ [4]. For both of these systems the blue phases appeared in the order BP I, BP II, BP III as the chiral fraction was increased, but it was the BP II phase that became unstable relative to BP I and BP III at even higher chirality.

We report here observations on the phase diagram of TOBMB using both polarizing microscopy and opticalactivity measurements. We undertook this study for two reasons. First, the TOBMB molecule is significantly different from the other molecules and has a much longer pitch. We suspected that this might be the reason why its phase diagram was reported to be different and were interested to find out if other properties, such as the chiral fluctuations in the isotropic phase, were also different. Second, a great deal has been learned recently about the blue phases so that phase identification is much easier, 
with other methods besides polarizing microscopy routinely being utilized to determine phase diagrams in highly chiral systems. Using all possible techniques to verify that the phase diagram of TOBMB was indeed different from other highly chiral liquid crystals struck us as an important task.

The TOBMB was prepared in both chiral and racemic forms starting from either $\mathrm{S}(-)$-2-methylbutan-1-ol or a racemic mixture of 2-methylbutan-1-ol [3]. Both the chiral and racemic forms melted around $137^{\circ} \mathrm{C}$ to a smectic- $A$ phase, underwent a transition to either a chiral-nematic or nematic phase at about $147^{\circ} \mathrm{C}$, and had an isotropic transition temperature just below $160^{\circ} \mathrm{C}$. The chiral version also possessed blue phases within $1^{\circ} \mathrm{C}$ of the isotropic transition. Since the optical activity in the isotropic phase just above the transition was positive, this chiral version of TOBMB was right handed. The fact that the optical activity in the blue phases was negative when the wavelength was longer than the pitch is also confirmation of a right-handed structure. The phase-transition temperatures were measured in an Instec Model HS1 hot stage using a Leitz Laborlux 12 Pol microscope, except for the $100 \%$ chiral sample, which was investigated using optical-activity measurements. The samples were either 25 or $50 \mu \mathrm{m}$ thick, and the temperature gradient across the illuminated part of the sample was less than $0.02 \mathrm{~K}$. All phase-transition temperatures were measured with increasing temperature. In the $100 \%$ chiral sample, the BP I to BP III transition was detected by a discontinuity in the optical activity, while the BP III to isotropic transition caused only a change in the temperature dependence of the optical activity (negative values, increasing rapidly toward zero in BP III; positive values, decreasing slowly in the isotropic phase). When samples with high chiral fraction were cooled, the supercooled BP I phase changed into another phase, which in turn changed into the chiral-nematic phase. This was probably the recently observed BP $S$ phase $[5,6]$. In addition, the presence of "ribbons" when heating from the smectic- $A$ phase to the chiral-nematic phase in all but the lowest chirality mixtures suggested the presence of a narrow twist-grain-boundary (TGB) $A$ phase $[7,8]$.

The phase diagram for TOBMB is shown in Fig. 1. It differs from the earlier reported phase diagram in two ways: (1) the temperature ranges of the blue phases are about $30 \%$ smaller, and (2) the BP II phase is not present at high chirality as opposed to the BP I phase. The second difference is crucial, so we sought additional evidence in support of this finding. It came in the observation that with extremely slow cooling, large, square, single crystals of BP II could be grown out of BP III. This is in fact the only way large, square, single crystals of BP II have been grown, as this does not occur when there is no BP III phase between the BP II and isotropic phases [9]. When only two blue phases were present at higher chiral fractions in TOBMB, the same procedure did not produce single crystals of the phase below BP III, indicating that the lower temperature phase was not BP II. Again, this is what has been found in another highly chiral system [10].

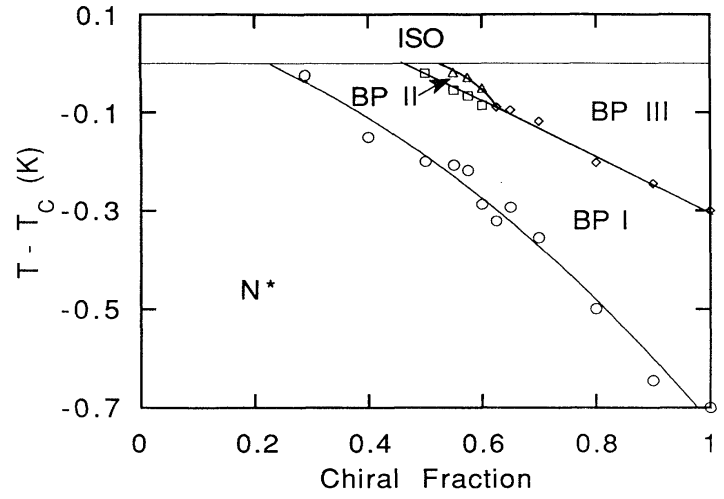

FIG. 1. Phase diagram of the blue phases for TOBMB. $T_{c}$ is the transition temperature to the isotropic phase at each concentration and is equal to $159.6 \pm 0.2 \mathrm{~K}$ for all mixtures. $O$, chiral-nematic-BP I transition; $\square$, BP I-BP II transition; $\diamond$, BP I-BP III transition; $\triangle$, BP II-BP III transition. The lines have been drawn to aid the eye. ISO and $N^{*}$ denote the isotropic and chiral-nematic phases, respectively.

With the present finding, all experimental phase diagrams for the blue phases of highly chiral systems have the same topology. In fact, even a casual look at them reveals that the chirality at which each blue phase appears and the rate at which the temperature range of each grows are proportionally quite similar. To test this further, the temperature and chirality axes of the phase diagrams for CE4 and CE2 from Ref. [4] were scaled to see if they could be made to agree with the TOBMB data. The data for the entire phase diagram were used in each case, and linear interpolation between data points was used to make numerical comparisons between phase diagrams. Multiplying all chiral fractions and temperatures for CE4 by 0.70 and 0.69 , respectively, gave the lowest

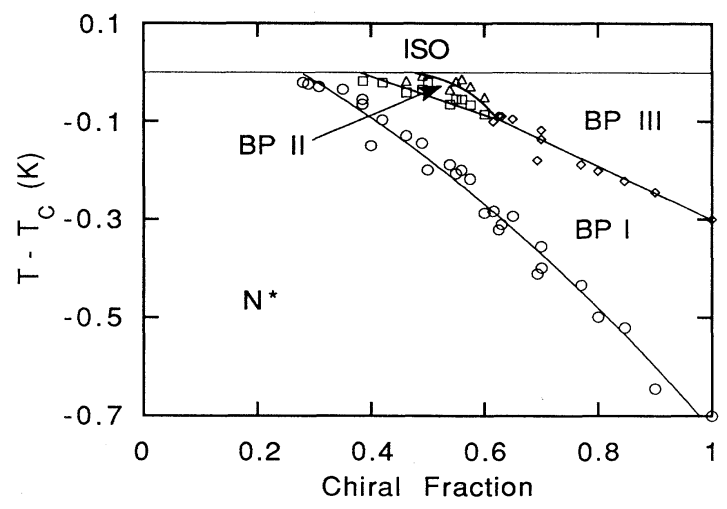

FIG. 2. Phase diagram of the blue phases for TOBMB and scaled phase diagrams for the blue phases of CE4 and CE2 (as described in the text). The CE4 and CE2 data are taken from Ref. [4]. $T_{c}$ is the transition temperature to the isotropic phase at each concentration for that compound. (O) chiralnematic-BP I transition; ( $\square$ ) BP I-BP II transition; ( $\diamond)$ BP I-BP III transition; and ( $\triangle$ ) BP II-BP III transition. The lines have been drawn to aid the eye. ISO and $N^{*}$ denote the isotropic and chiral-nematic phases, respectively. 
sum of the squares of the differences with the TOBMB data. Similarly, multiplying the chiral fractions and temperatures for CE2 by 1.54 and 0.59 , respectively, gave the lowest sum of the squares of the differences with the TOBMB data. The results of these two scalings are shown in Fig. 2, where the scaled CE4 and CE2 data are superimposed on the TOBMB data. The fact that a single pair of scalings makes one entire phase diagram quantitatively similar to another entire phase diagram for these three systems is at the very least surprising. After all, each compound is different in structure, number of chiral centers, and transition temperatures, and the free energy of all of the blue phases is extremely similar. Yet all of the phase diagrams are both qualitatively and quantitatively similar.

The numbers used in the scaling illustrate an important caveat to any discussion of a universal phase diagram of the blue phases. The pitch of the chiral-nematic phase at the transition to BP I is roughly $250 \mathrm{~nm}$ in TOBMB [3], $160 \mathrm{~nm}$ in CE4 [4], and $110 \mathrm{~nm}$ in CE2 [4]. Yet the chiral-fraction axis for CE4 must be contracted to agree with TOBMB, and the chiral-fraction axis for CE2 must be expanded to agree with TOBMB, even though both have significantly shorter pitch. Meanwhile, the temper- ature axes for both CE4 and CE2 must be contracted by about the same amount to agree with TOBMB. These results clearly reflect the complex dependence of macroscopic properties on the details of the intermolecular interactions, as has been suspected all along. Yet in spite of this complexity, the phase diagrams of the blue phases seem to show significant similarities.

There may be some simple theoretical ideas combining thermodynamics and symmetry that argue for a universal phase diagram for the blue phases. While the experimental evidence for such is not very strong at this time due to the small number of systems studied, the fact that any lack of consistency between the data points of Fig. 2 is probably within experimental error makes one wonder if there is something extremely important about this result. Further theoretical work, as well as the experimental investigation of more highly chiral systems, is certainly warranted.

These results are based upon work supported by the National Science Foundation under Grant No. DMR9196048 and the W. M. Keck Foundation. The authors would like to thank P. P. Crooker for supplying us with the original data on the phase diagrams of CE4 and CE2.
[1] D. C. Wright and N. D. Mermin, Rev. Mod. Phys. 61, 385 (1989).

[2] T. Seideman, Rep. Prog. Phys. 53, 659 (1990).

[3] M. A. Marcus and J. W. Goodby, Mol. Cryst. Liq. Cryst. 72, 297 (1982).

[4] D. K. Yang and P. P. Crooker, Phys. Rev. A 35, 4419 (1987).

[5] E. Demikhov and H. Stegemeyer, Liq. Cryst. 10, 869 (1991).

[6] E. Demikhov, H. Stegemeyer, and V. Tsukruk, Phys. Rev.
A 46, 4879 (1992).

[7] J. W. Goodby, M. A. Waugh, S. M. Stein, E. Chin, R. Pindak, and J. S. Patel, J. Am. Chem. Soc. 111, 8119 (1989).

[8] J. W. Goodby, M. A. Waugh, S. M. Stein, E. Chin, R. Pindak, and J. S. Patel, Nature (London) 377, 449 (1989).

[9] H. Stegemeyer, Th. Blumel, K. Hiltrop, H. Onusseit, and F. Porsch, Liq. Cryst. 1, 3 (1986).

[10] J. D. Miller, P. R. Battle, P. J. Collings, D. K. Yang, and P. P. Crooker, Phys. Rev. A 35, 3959 (1987). 